\title{
Caractérisation électrique de couches minces conductrices par une méthode hyperfréquence sans contact
}

\author{
X. Le Cleac'h, M. Bellec \\ Laboratoire de Physique des Matériaux, Institut Universitaire de Technologie, \\ Université de Rennes, 22302 Lannion, France
}

et G. Grandpierre

Département M.P.A., C.N.E.T., 22300 Lannion, France

(Reçu le 23 décembre 1981, révisé le 26 avril 1982, accepté le 19 mai 1982)

\begin{abstract}
Résumé. - La méthode des cavités hyperfréquence faiblement perturbées est utilisée pour la caractérisation électrique, c'est-à-dire la détermination de la mobilité de Hall et du nombre de porteurs libres de couches minces conductrices créées à partir de substrats massifs isolants. La mesure de la mobilité $\mu$ est effectuée à $14 \mathrm{GHz}$ à l'aide d'une cavité cylindrique bimodale où seulement deux modes TE 111 dégénérés sont excités. La conductivité $\sigma$ est mesurée à deux fréquences différentes : $14 \mathrm{GHz}$ et $560 \mathrm{MHz}$ (cavité coaxiale réentrante). Cette précaution permet, dans le cas où les couches sont très conductrices, de diminuer l'erreur due à la valeur mal connue du facteur de dépolarisation des substrats et, dans le cas où elles sont isolantes, de déceler l'intervention éventuelle d'un mécanisme de conduction autre que celui dû aux porteurs libres qui est indépendant de la fréquence $(\omega \tau \ll 1)$. Le domaine d'utilisation des montages proposés n'est limité que par la sensibilité des systèmes de détection vers les basses valeurs de $\sigma$ et $\mu$ et par l'amplitude de la perturbation vers les hautes valeurs pour des couches minces dont l'épaisseur est voisine du micron, c'est-à-dire bien inférieure à la profondeur d'effet de peau. L'accord est excellent entre nos résultats obtenus avec contact électrique en continu et sans contact en hyperfréquence.
\end{abstract}

\begin{abstract}
Cavity perturbation method is used for the measurement of mobility $\mu$ and electron carrier concentration $n$ of conducting thin films made on bulk insulating substrates. The microwave mobility is measured at $14 \mathrm{GHz}$ with a bimodal cylindrical cavity where only two TE 111 degenerate modes are excited. Conductivity $(\sigma)$ measurements are made at $14 \mathrm{GHz}$ and $560 \mathrm{MHz}$ (re-entrant coaxial cavity). This precaution permits, for very conducting films, to reduce the error arising from the imprecise value of the substrate depolarization factor, and for insulating films, to disclose the eventual presence of another mechanism of conduction depending on frequency $(\omega \tau \sim 1)$. The measurement range of these different cavities is limited only by the sensibility of detectors toward low values of $\sigma$ and $\mu$ and by the magnitude of the perturbation toward high values for thin films which thickness equals about one micron, that it to say, most inferior to the skin effect deepness. The concordance is good between d.c. (with electrical contacts) and microwave (without contact) experimental results.
\end{abstract}

1. Introduction. - Depuis fort longtemps, les techniques hyperfréquences sont utilisées pour effectuer des mesures sans contact électrique sur les matériaux susceptibles d'être employés en électronique; c'est le cas en particulier des diélectriques [1, 2]. Elles sont également d'un grand intérêt pour l'étude de différents phénomènes physiques intervenant dans les matériaux en général (rotation Faraday [3], conduction par sauts d'électrons dans les semi-conducteurs [4],...) ou bien, tout simplement, pour la caractérisation électrique d'un matériau de dimensions si petites qu'il est impossible de déposer des contacts pour des mesures en continu [5].
Le problème présent est d'étudier des couches minces conductrices créées à partir de substrats isolants, qui sont généralement sous forme de plaquettes de $5 \times 5 \mathrm{~mm}^{2}$ environ. La caractérisation, c'est-à-dire la détermination du nombre de porteurs libres et de la mobilité de Hall, doit être non destructive pour qu'elles puissent être réutilisées pour d'autres expériences. Notons également qu'il est parfois difficile d'effectuer la même caractérisation en continu car, pour un matériau nouveau, il faut trouver la bonne solution pour réaliser le bon contact ohmique dès le premier essai si possible. Notre chaîne de mesure, utilisant des cavités faiblement perturbées fonction- 
nant à $560 \mathrm{MHz}$ et $14 \mathrm{GHz}$, permet d'éviter ces différentes difficultés. Il existe d'autres méthodes basées sur l'absorption de puissance par courants de Foucault $[6,7]$ permettant également des mesures non destructives des échantillons mais ne donnant qu'un seul paramètre, la conductivité.

La première partie de cet article est consacrée, d'une part, aux problèmes de mesure de la conductivité, ou plus précisément à ceux provenant des différents champs électriques de dépolarisation, en particulier à celui dû aux porteurs libres, et d'autre part à la nécessité de travailler à deux fréquences différentes. $\mathrm{La}$ méthode originale de mesure à $560 \mathrm{MHz}$ par cavité coaxiale est décrite. La partie suivante traite de la technique de mesure de $\mu_{\mathrm{H}}$ à $14 \mathrm{GHz}$, des conditions de réglage et d'utilisation indispensables pour un bon fonctionnement, ainsi que de la méthode particulière utilisée pour les matériaux très peu perturbants. La dernière partie est consacrée aux résultats expérimentaux.

2. Conductivité. - 2.1 Méthode de CALCUl. La technique de mesure utilisée est celle des cavités faiblement perturbées fonctionnant dans le domaine des hyperfréquences. L'échantillon étant placé parallèlement au champ électrique, les paramètres de la conductivité sont déterminés par la relation suivante [8] :

$$
2\left(\frac{F_{1}-F_{0}}{F_{0}}\right)-i\left(\frac{1}{Q_{1}}-\frac{1}{Q_{0}}\right)=(\varepsilon-1) \frac{\int_{v} E_{1} E_{0} \mathrm{~d} v}{\int_{V} E_{0}^{2} \mathrm{~d} V}
$$

où $F_{1}$ et $F_{0}, Q_{1}$ et $Q_{0}$ sont les fréquences de résonance et les coefficients de qualité de la cavité avec et sans échantillon, $\varepsilon=\varepsilon^{\prime}-i \varepsilon^{\prime \prime}$ la constante diélectrique relative complexe, $v$ le volume de l'échantillon, $V$ celui de la cavité, $E_{0}$ le champ électrique dans la cavité à vide et $E_{1}$ celui existant à l'intérieur de l'échantillon.

La théorie des petites perturbations implique que $E_{0}$ n'est pas modifié dans tout le volume de la cavité par la présence de l'échantillon à étudier, sauf à l'intérieur de celui-ci où $E_{1}$ est donné par la relation suivante [9] :

$$
E_{1}=\frac{E}{1+L\left(\varepsilon^{\prime}-1-i \varepsilon^{\prime \prime}\right)}
$$

où $L$ est un facteur de dépolarisation et $E$ la valeur de $E_{0}$ à l'emplacement de l'échantillon quand celui-ci est sorti de la cavité.

En plus du champ dépolarisant dû à la constante diélectrique $\varepsilon^{\prime}$ du matériau, il faut tenir compte d'un autre, que nous appellerons $E_{\mathrm{d}}$ et qui est dû au déplacement des porteurs libres :

$$
E_{\mathrm{d}} \propto n e r
$$

où $n$ est le nombre de porteurs par centimètre cube, $e$ la charge de l'électron et $r$ la distance entre le centre de gravité des charges des atomes ionisés et celui des électrons de conduction. La dépolarisation correspondant à $E_{\mathrm{d}}$ est représentée par $L \varepsilon^{\prime \prime}$ dans la formule (2).

Les équations (1) et (2) sont valables pour un matériau isotrope alors que notre problème actuel est d'étudier des couches minces obtenues à partir d'un substrat semi-isolant, soit par implantation ionique [11], soit par jet moléculaire [12]. Le matériau à étudier se compose donc de deux parties (Fig. 1) :

1. Le substrat semi-isolant massif dont l'épaisseur est généralement supérieure à $200 \mu$. Soient $\varepsilon_{1}=\varepsilon_{1}^{\prime}-i \varepsilon_{1}^{\prime \prime}$ et $L_{1}$ respectivement sa constante diélectrique et son facteur de dépolarisation.

2. La couche mince, d'épaisseur voisine d'un micron, de constante diélectrique $\varepsilon_{2}=\varepsilon_{2}^{\prime}-i \varepsilon_{2}^{\prime \prime}$ et de facteur de dépolarisation $L_{2}$.

Le problème peut être traité de façon générale mais nous ne discuterons que de notre cas particulier :

$$
\varepsilon_{1}^{\prime} \sim \varepsilon_{2}^{\prime} ; \quad \varepsilon_{2}^{\prime \prime} \gg \varepsilon_{1}^{\prime} \gg \varepsilon_{1}^{\prime \prime} \quad \text { et } \quad L_{1} \gg L_{2} .
$$

Cette dernière propriété s'explique par le fait que, pour des ellipsoïdes très plats, $L$ est pratiquement proportionnel à l'épaisseur. Le second membre de l'équation (1) devient donc :

$$
\begin{array}{r}
{\left[\frac{\left(\varepsilon_{1}^{\prime}-1\right) \int_{v_{1}} E_{0}^{2} \mathrm{~d} v}{1+L_{1}\left(\varepsilon_{1}^{\prime}-1\right)}+\frac{\left(\varepsilon_{2}^{\prime}-1-i \varepsilon_{2}^{\prime \prime}\right) \int_{v_{2}} E_{0}^{2} \mathrm{~d} v}{\left[1+L_{1}\left(\varepsilon_{1}^{\prime}-1\right)-i L_{2} \varepsilon_{2}^{\prime \prime}\right]}\right] \times} \\
\times \frac{1}{\int_{V} E_{0}^{2} \mathrm{~d} V}
\end{array}
$$

où $v_{1}$ et $v_{2}$ sont respectivement les volumes du substrat et de la couche mince avec $v_{1} \gg v_{2}$.

On considère généralement que le champ est constant dans le volume qu'occupe l'échantillon, c'est-à-dire que les dimensions de celui-ci sont petites par rapport à la longueur d'onde du champ électromagnétique. Dans la région 1 de l'échantillon, le champ est donné par l'expression :

$$
\frac{E}{1+L_{1}\left(\varepsilon_{1}^{\prime}-1\right)}
$$

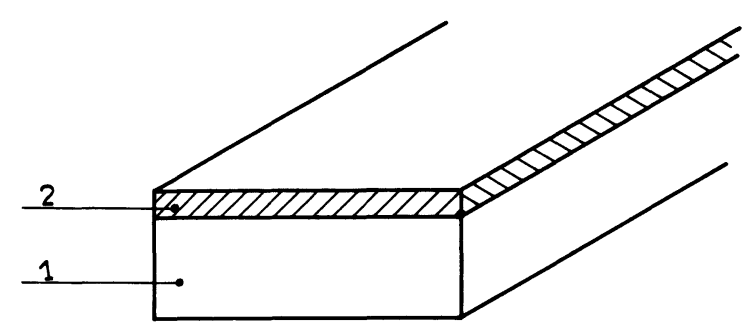

Fig. 1. - Echantillon à étudier : 1. Substrat isolant ; 2. Couche mince conductrice.

[Sample : 1 . Insulating substrate $; 2$. Conducting thin film.] 
et, pour la région $\underline{2}$, par :

$$
\frac{E}{\left[1+L_{1}\left(\varepsilon_{1}^{\prime}-1\right)-i L_{2} \varepsilon_{2}^{\prime \prime}\right]} \text {. }
$$

En posant :

$$
\frac{E^{2}}{\int_{V} E_{0}^{2} \mathrm{~d} V}=H
$$

l'expression (3) peut s'écrire :

$$
\begin{aligned}
& 2\left(\frac{F_{1}-F_{0}}{F_{0}}\right)-i\left(\frac{1}{Q_{1}}-\frac{1}{Q_{0}}\right)= \\
& \quad=\left\lceil\frac{\left(\varepsilon_{1}^{\prime}-1\right) v_{1}}{1+L_{1}\left(\varepsilon_{1}^{\prime}-1\right)}-\frac{i \varepsilon_{2}^{\prime \prime} v_{2}}{\left[1+L_{1}\left(\varepsilon_{1}^{\prime}-1\right)-i L_{2} \varepsilon_{2}^{\prime \prime}\right]}\right\rceil \times H .
\end{aligned}
$$

Pour des échantillons à champ dépolarisant $E_{\mathrm{d}}$ négligeable, nous pouvons écrire :

$$
\left.\begin{array}{c}
\frac{F_{1}-F_{0}}{F_{0}}=\frac{1}{2} \frac{\left(\varepsilon_{1}^{\prime}-1\right) v_{1}}{1+L_{1}\left(\varepsilon_{1}^{\prime}-1\right)} H \\
\left(\frac{1}{Q_{1}}-\frac{1}{Q_{0}}\right)=\frac{\varepsilon_{2}^{\prime \prime} v_{2}}{1+L_{1}\left(\varepsilon_{1}^{\prime}-1\right)} H \\
\text { ou : }\left(\frac{1}{Q_{0}}-\frac{1}{Q_{1}}\right) \times \frac{F_{0}}{F_{0}-F_{1}}=\frac{2 \varepsilon_{2}^{\prime \prime} v_{2}}{\left(\varepsilon_{1}^{\prime}-1\right) v_{1}} .
\end{array}\right\}
$$

Rappelons que ces équations ne sont utilisables que si $\varepsilon_{2}^{\prime}$ est voisin de $\varepsilon_{1}^{\prime}$. La condition « $L_{2} \varepsilon_{2}^{\prime \prime}$ négligeable » est vérifiée si les valeurs des conductivités obtenues à deux fréquences différentes (dans le cas présent : $560 \mathrm{MHz}$ et $14 \mathrm{GHz}$ ) sont identiques et si la dérive en fréquence correspond bien aux paramètres $\varepsilon_{1}^{\prime}, v_{1}$ et $L_{1}$.

Dans le cas où le champ dépolarisant n'est plus négligeable, on peut déduire l'expression de $\varepsilon_{2}^{\prime \prime}$ de l'équation (7) :

$$
\varepsilon_{2}^{\prime \prime}=\frac{A \Delta}{v_{2}}\left[1+\left(\frac{\delta-\left(\varepsilon_{1}^{\prime}-1\right) v_{1} / A}{\Delta}\right)^{2}\right]
$$

avec :

$$
\begin{aligned}
\Delta & =\left(\frac{1}{Q_{1}}-\frac{1}{Q_{0}}\right) \times \frac{1}{H} \\
\delta & =2\left(\frac{F_{1}-F_{0}}{F_{0}}\right) \times \frac{1}{H} \\
A & =1+L_{1}\left(\varepsilon_{1}^{\prime}-1\right) .
\end{aligned}
$$

On a aussi :

$$
L_{2} \varepsilon_{2}^{\prime \prime}=\frac{A \delta-\left(\varepsilon_{1}^{\prime}-1\right) v_{1}}{A \Delta} .
$$

Ici, le terme mal connu est $A$, ou plus précisément $L_{1}$ le facteur de dépolarisation calculé pour des ellip- soïdes et non des plaquettes. Il a une grosse influence quand $\left(\varepsilon_{1}^{\prime}-1\right) v_{1}$ devient du même ordre de grandeur que $A \delta$ et peut entraîner une grosse erreur. En pratique, cela correspond à des valeurs de $L_{2} \varepsilon_{2}^{\prime \prime}$ voisines de 1 . Il est alors préférable de travailler à $560 \mathrm{MHz}$ où $L_{2} \varepsilon_{2}^{\prime \prime}$ est multiplié par 25. L'erreur relative sur $\varepsilon_{2}^{\prime \prime}$ devient alors voisine de celle existant sur $A$.

2.2 MONTAGeS EXPÉRIMENTAUX. - Les mesures de conductivité à $14 \mathrm{GHz}$ ont été effectuées à l'aide d'une cavité rectangulaire fonctionnant dans le mode TE 105 [13] en transmission. L'échantillon est positionné au centre de la cavité, là où la distribution du champ électrique passe par un maximum. Il est indispensable de faire les mesures à vide et avec échantillon sans un démontage mécanique de la cavité, une telle opération risquerait d'entraîner des modifications de la fréquence de résonance et du facteur de qualité. Les échantillons se présentant sous la forme de plaquettes de $5 \times 5 \times 0,4 \mathrm{~mm}^{3}$ environ, leur introduction se fait par une fente effectuée par électro-érosion dans l'une des parois de la cavité et coïncidant avec une ligne de courant électrique du mode considéré (Fig. 2). Cette rainure n'apporte qu'une perturbation très minime, une dérive de $10 \mathrm{MHz}$ environ de la fréquence de résonance de la cavité. L'échantillon est maintenu par un support en poly-

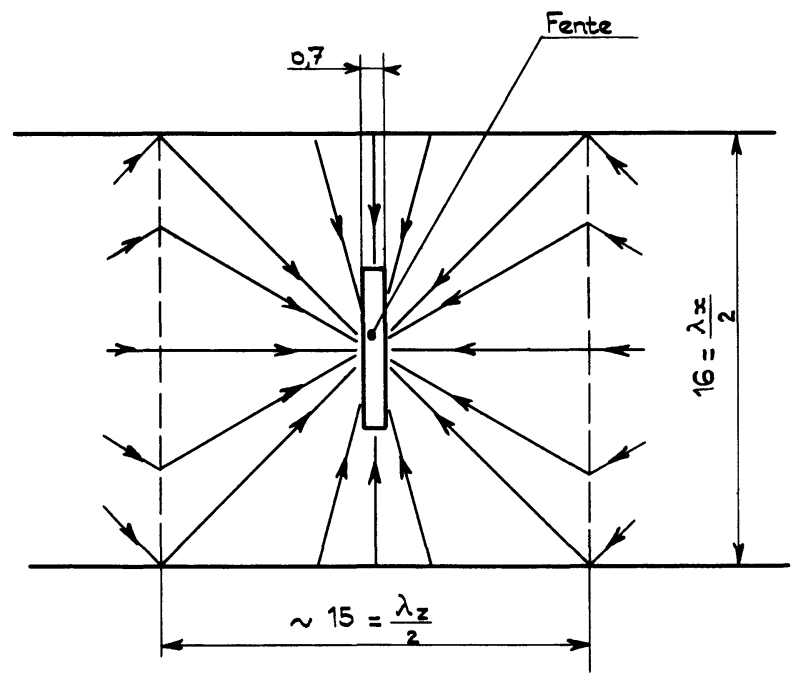

Fig. 2. - Position de la fente par rapport à la distribution des lignes de courant électrique dans la paroi de la cavité rectangulaire aux alentours d'un maximum de champ électrique. Dans cette région, le courant passe par un minimum et la fente, disposée parallèlement à l'une des lignes de courant, crée une perturbation négligeable. Les dimensions sont données en millimètres. $\lambda_{x}$ et $\lambda_{z}$ sont les longueurs d'onde apparentes suivant deux axes de la cavité dans le mode TE $10 \mathrm{n}$.

[Position of the split with regard to the distribution of electric current lines in a region where electric field is maximum. The split is parallel to the nearest current lines, the current is zero at the centre. Cavity perturbation is negligible. Dimensions are given in millimetres. $\lambda_{x}$ and $\lambda_{z}$ are the apparent wavelengths in the cavity working in the TE $10 \mathrm{n}$ mode.] 


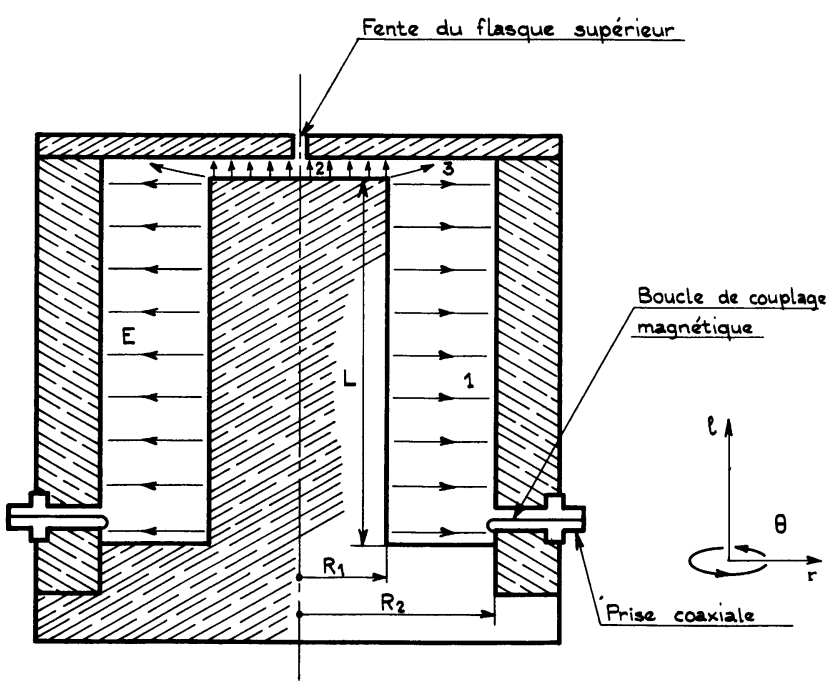

Fig. 3. - Vue schématique en coupe d'une cavité coaxiale avec la distribution du champ électrique.

[Schematic cross-section of the coaxial cavity with its electric field distribution.]

styrène de faibles pertes. Le terme inconnu, $H$, des équations (8), (10) et (11) peut être aisément calculé, connaissant la distribution du champ électrique [13] :

$$
H=\frac{4}{V} \text {. }
$$

Pour nos mesures à $560 \mathrm{MHz}$, nous avons utilisé une cavité coaxiale travaillant en transmission. La figure 3 en présente une vue en coupe. L'excitation s'effectue par des boucles de couplage magnétique placées là où le champ magnétique est maximum. Comme pour la cavité rectangulaire, la conductivité se déduit des variations du coefficient de qualité et de la fréquence de résonance à vide et avec échantillon.

Le calcul de $H$ peut se déduire de la distribution du champ électrique qui est connue [14] pour les régions $\underline{1}$ et 2 :

$$
\begin{aligned}
& E_{\mathrm{C}_{1}}=\frac{D}{r} \sin 2 \pi \frac{l}{\lambda} \\
& E_{\mathrm{C}_{2}}=\frac{D}{d} \log \frac{R_{2}}{R_{1}} \sin 2 \pi \frac{L}{\lambda}
\end{aligned}
$$

où $r$ est la distance entre le point considéré et l'axe de symétrie, $l$ la hauteur au-dessus du plan courtcircuit, $\lambda$ la longueur d'onde, $D$ une constante, $R_{1}$ et $R_{2}$ les rayons intérieur et extérieur de la partie coaxiale, $d$ la distance entre l'extrémité de l'âme centrale de la cavité et le flasque supérieur, et $L$ la longueur de l'âme centrale.

Par contre, la région $\underline{3}$, où les lignes de force sont mal connues, est négligée pour le calcul de $H$ à l'aide de l'équation (6). Ceci peut être justifié. Au déno-

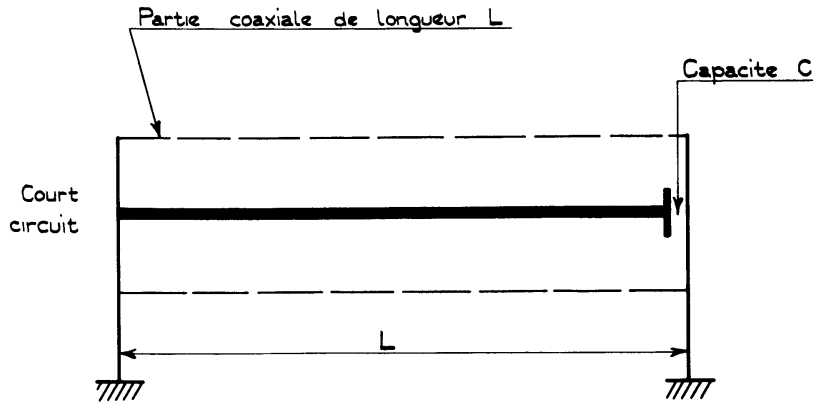

Fig. 4. - Schéma électrique équivalent de la cavité coaxiale.

[Equivalent network of the reentrant coaxial cavity.]

minateur de (6), intervient l'intégrale $\int_{V} E_{0}^{2} \mathrm{~d} v$ qui est proportionnelle à l'énergie emmagasinée à une constante près $\varepsilon_{0}$; et pour les régions 2 et 3 , cette énergie peut être représentée par $\frac{1}{2} C V^{2}$, où $C$ est la capacité en bout de ligne et $V$ la différence de potentiel à ses bornes :

$$
V=D \log \frac{R_{2}}{R_{1}} \sin 2 \pi \frac{L}{\lambda} .
$$

Pour une raison de géométrie, la capacité $C$, et donc l'énergie, est principalement localisée en 2 . Ceci peut être confirmé expérimentalement. Soit la cavité représentée par son schéma équivalent (Fig. 4) composé d'une ligne coaxiale de longueur $L$ court-circuitée à une extrémité et fermée sur une capacité $C$ à l'autre, la condition de résonance est donnée par la relation :

$$
\frac{1}{Z_{0} C 2 \pi F}=\operatorname{tg} 2 \pi \frac{L}{\lambda}
$$

où $Z_{0}$ est l'impédance caractéristique de la ligne et $F$ la fréquence de résonance. $\mathrm{La}$ valeur de $C$, déduite expérimentalement à l'aide de (18), est supérieure de $10 \%$ à celle du condensateur constituant uniquement la région 2. En fait, il faut tenir compte de l'énergie emmagasinée dans la région 1 et on peut estimer que pour les dimensions de notre cavité, l'erreur sur $H$ est inférieure à $3 \%$ si on néglige la région 3.

L'échantillon à mesurer est placé en 2 , sans contact avec les parois métalliques de la cavité, parallèlement au champ électrique qui est uniforme dans cette région. Comme pour la cavité rectangulaire, l'introduction des plaquettes se fait par une fente effectuée dans le flasque supérieur (Fig. 3).

Des équations (1), (15) et (16), on déduit $\varepsilon_{2}^{\prime \prime}$ dans le cas où le champ dépolarisant dû aux porteurs libres est négligé : 


$$
\varepsilon_{2}^{\prime \prime}=\left(\frac{1}{Q_{1}}-\frac{1}{Q_{0}}\right) \frac{\pi d^{2}\left[L-\frac{\sin 2 \beta L}{2 \beta}+\frac{R_{1}^{2}}{d} \sin ^{2} \beta L \log \frac{R_{2}}{R_{1}}\right]}{v_{2}\left(\log \frac{R_{2}}{R_{1}} \sin ^{2} \beta L\right)}\left[1+L_{1}\left(\varepsilon_{1}^{\prime}-1\right)\right]
$$

où $\beta$ vaut $2 \pi / \lambda$.

Avec $d=0,4 \mathrm{~cm}, L=11 \mathrm{~cm}, R_{1}=2 \mathrm{~cm}, R_{2}=3 \mathrm{~cm}$, $F=560 \mathrm{MHz}$, on obtient dans le système M.K.S.A. :

$$
\varepsilon_{2}^{\prime \prime} \approx 16,8 \times 10^{-6}\left(\frac{1}{Q_{1}}-\frac{1}{Q_{0}}\right) \frac{1+L_{1}\left(\varepsilon_{1}^{\prime}-1\right)}{v_{2}} .
$$

Dans le cas où le champ dépolarisant n'est plus négligeable, il faut utiliser les équations (9) à (13) avec :

$$
H=6,6 \times 10^{4}
$$

(système M.K.S.A.).

3. Mobilité. - 3.1 Principe de Mesure. - La mesure de mobilité de Hall en hyperfréquence peut s'effectuer à l'aide de cavités dont la géométrie permet l'existence de deux modes orthogonaux de même fréquence de résonance. Ainsi, pour une cavité parallélépipédique de section carrée, les deux modes TE $10 \mathrm{n}$ et TE $01 \mathrm{n}$ peuvent exister simultanément [15] ; quand la cavité est cylindrique, le mode TE $11 \mathrm{n}$ peut être créé théoriquement suivant n'importe quelle direction.

En l'absence d'échantillon, le premier mode, ou plus précisément son orientation, est défini par la nature du couplage électrique ou magnétique entre la cavité et les connecteurs de liaison (guide rectangulaire ou câble coaxial) avec la source hyperfréquence. $\mathrm{Si}$ un matériau est placé parallèlement au champ électrique, un champ magnétique continu induit un deuxième champ électrique orthogonal au premier et de même fréquence. Ceci implique bien sûr qu'il y ait une géométrie absolument identique de la cavité et de l'échantillon à mesurer suivant les deux directions orthogonales.

Sur le plan théorique, le problème a été longuement développé par Watanabe [16]. Son calcul, basé sur l'existence de deux modes doublement dégénérés et sur les travaux correspondants de Slater [17] concernant les cavités résonnantes, aboutit à la matrice de transmission en paramètres $S$ d'une cavité avec deux entrées ou sorties possibles positionnées à 90 degrés l'une de l'autre (Fig. 5a).

$$
\left[\begin{array}{l}
b_{1} \\
b_{2}
\end{array}\right]=\left[\begin{array}{ll}
S_{11} & S_{12} \\
S_{21} & S_{22}
\end{array}\right]\left[\begin{array}{l}
c_{1} \\
c_{2}
\end{array}\right]
$$

où $b_{1}$ et $b_{2} c_{1}$ et $c_{2}$ sont les amplitudes des ondes réfléchies ou incidentes en 1 et $2 ; S_{11}$ et $S_{22}$ les coef-

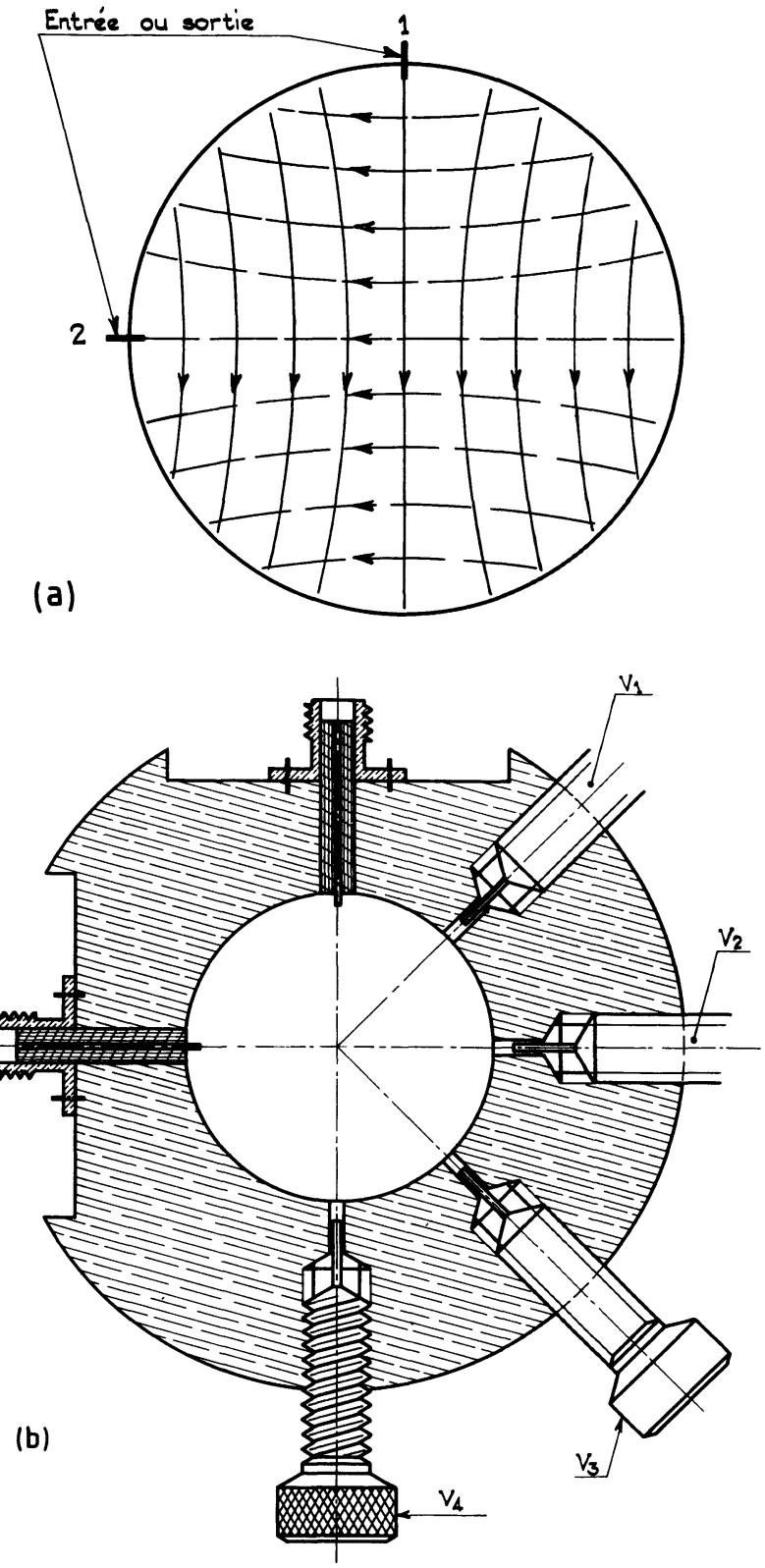

Fig. 5. - a) Distributions du champ électrique des deux modes orthogonaux en TE $11 \mathrm{n}$ dans une cavité cylindrique. b) Vue schématique en coupe montrant les vis de réglage de la cavité.

[a) Electrical field distributions of the two TE $11 \mathrm{n}$ orthogonal modes in a cylindrical cavity. b) Schematic crosssection of the cavity pointing out the tuning screwes.]

ficients de réflexion en 1 et $2 ; S_{12}$ et $S_{21}$ les coefficients de transmission de 1 à 2 , et vice versa, tels que : 


$$
\begin{aligned}
S_{11}= & \frac{\left[\frac{1}{Q x_{1}}-\left(\frac{1}{Q_{1}}-\frac{1}{Q_{0}}\right)-\frac{1}{Q w}\right]}{\left[\frac{1}{Q x_{1}}+\left(\frac{1}{Q_{1}}-\frac{1}{Q_{0}}\right)+\frac{1}{Q w}\right]} \\
\left(\frac{P_{2}}{P_{1}}\right)^{1 / 2}=S_{12} & =\frac{\left[\frac{2\left|\sigma_{1}\right|}{\operatorname{Re}\left(\sigma_{0}\right)}\left(\frac{1}{Q_{1}}-\frac{1}{Q_{0}}\right)\left(Q x_{1} \times Q x_{2}\right)^{-1 / 2}\right]}{\left[\frac{1}{Q x_{1}}+\frac{1}{Q w}+\left(\frac{1}{Q_{1}}-\frac{1}{Q_{0}}\right)\right]\left[\frac{1}{Q x_{2}}+\frac{1}{Q w}+\left(\frac{1}{Q_{1}}-\frac{1}{Q_{0}}\right)\right]}=-S_{21} \\
S_{22} & =\frac{\left[\frac{1}{Q x_{2}}-\frac{1}{Q w}-\left(\frac{1}{Q_{1}}-\frac{1}{Q_{0}}\right)\right]}{\left[\frac{1}{Q x_{2}}+\frac{1}{Q w}+\left(\frac{1}{Q_{1}}-\frac{1}{Q_{0}}\right)\right]}
\end{aligned}
$$

où :

$$
\mu B=\frac{\left|\sigma_{1}\right|}{\operatorname{Re}\left(\sigma_{0}\right)}
$$

$\mu$ étant la mobilité, $B$ le champ magnétique, $\sigma_{0}$ et $\sigma_{1}$ les composantes longitudinale et transversale du tenseur de conductivité de l'échantillon, $P_{1}$ et $P_{2}$ les puissances incidente en 1 et transmise en 2, $Q x_{1}$ et $Q x_{2}$ les facteurs de qualité correspondant au couplage entre les modes de résonance et chacune des entrées, $Q w$ le facteur de qualité correspondant aux pertes dans les parois de la cavité.

Les équations (23) à (26) sont valables, à champ magnétique faible, pour des échantillons dont l'épaisseur est nettement inférieure à la profondeur d'effet de peau, n'ayant aucun contact avec les parois métalliques et placés parallèlement au champ électrique sur l'axe de symétrie de la cavité. Les échantillons eux-mêmes doivent être symétriques pour avoir des facteurs de dépolarisation identiques suivant les deux directions des modes orthogonaux. Le champ dépolarisant dû aux porteurs libres n'intervient pas dans l'équation donnant la mobilité, selon Watanabe [18].

Sachant que $S_{11}$ et $S_{22}$ sont les coefficients de réflexion en 1 et 2, l'équation (24) peut se mettre sous la forme suivante [19] :

$$
\mu=\frac{\left[\left(1+\Gamma_{10}\right)\left(1+\Gamma_{20}\right)\right]^{1 / 2}}{B\left[\left(1+\Gamma_{11}\right)\left(1+\Gamma_{21}\right)\left(\Gamma_{11}-\Gamma_{10}\right)\left(\Gamma_{21}-\Gamma_{20}\right)\right]^{1 / 2}}\left(\frac{P_{2}}{P_{1}}\right)^{1 / 2}
$$

où $\Gamma_{p q}$ est le coefficient de réflexion, avec $p$ prenant les valeurs 1 ou 2 selon l'entrée concernée et $q$ les valeurs 0 ou 1 selon que la cavité est vide ou chargée. Le coefficient de réflexion est positif ou négatif selon que la cavité est surcouplée ou sous-couplée, et nul au couplage critique.

Dans le cas de symétrie de la matrice $S\left(S_{11}=S_{22}\right.$ ou $\Gamma_{10}=\Gamma_{20}=\Gamma_{0}$ et $\Gamma_{11}=\Gamma_{21}=\Gamma_{1}$ ), c'est-à-dire un couplage identique pour chaque entrée, une symétrie parfaite de la cavité et de l'échantillon, l'équation précédente devient :

$$
\mu=\frac{\left(1+\Gamma_{0}\right)}{B\left(1+\Gamma_{1}\right)\left(\Gamma_{1}-\Gamma_{0}\right)}\left(\frac{P_{2}}{P_{1}}\right)^{1 / 2} .
$$

Pour la mesure de la mobilité, il n'est pas possible d'introduire l'échantillon sans démontage de la cavité par une fente coïncidant avec une ligne de courant dans une paroi métallique. Ceci s'explique par le fait que l'effet Hall fait intervenir deux modes orthogonaux auxquels correspondent deux distributions de lignes de courant électrique orthogonales. L'une ou l'autre de ces distributions sera obligatoirement coupée perpendiculairement par la fente et le mode correspondant sera fortement perturbé.

Quañ le matériau à mesurer est très isolant (faible perturbation), les démontage et remontage de la cavité peuvent entraîner des modifications de $Q_{1}$ et $F_{1}$ du même ordre de grandeur que celles provenant de l'échantillon. Dans ce cas, il est préférable d'opérer différemment. L'échantillon étant à l'intérieur de la cavité, les mesures de $\Gamma_{1}, Q_{1}, P_{2}$ et $P_{1}$ sont effectuées de façon habituelle [20]. On sait que la variation du facteur de qualité est donnée par la relation :

$$
\begin{aligned}
\left(\frac{1}{Q_{1}}-\frac{1}{Q_{0}}\right) \times \frac{1}{H}= \\
\quad=\frac{\left[1+L_{1}\left(\varepsilon_{1}^{\prime}-1\right)\right] \varepsilon_{2}^{\prime \prime} v_{2}}{\left[1+L_{1}\left(\varepsilon_{1}^{\prime}-1\right)\right]^{2}+\left(L_{2} \varepsilon_{2}^{\prime \prime}\right)^{2}}
\end{aligned}
$$




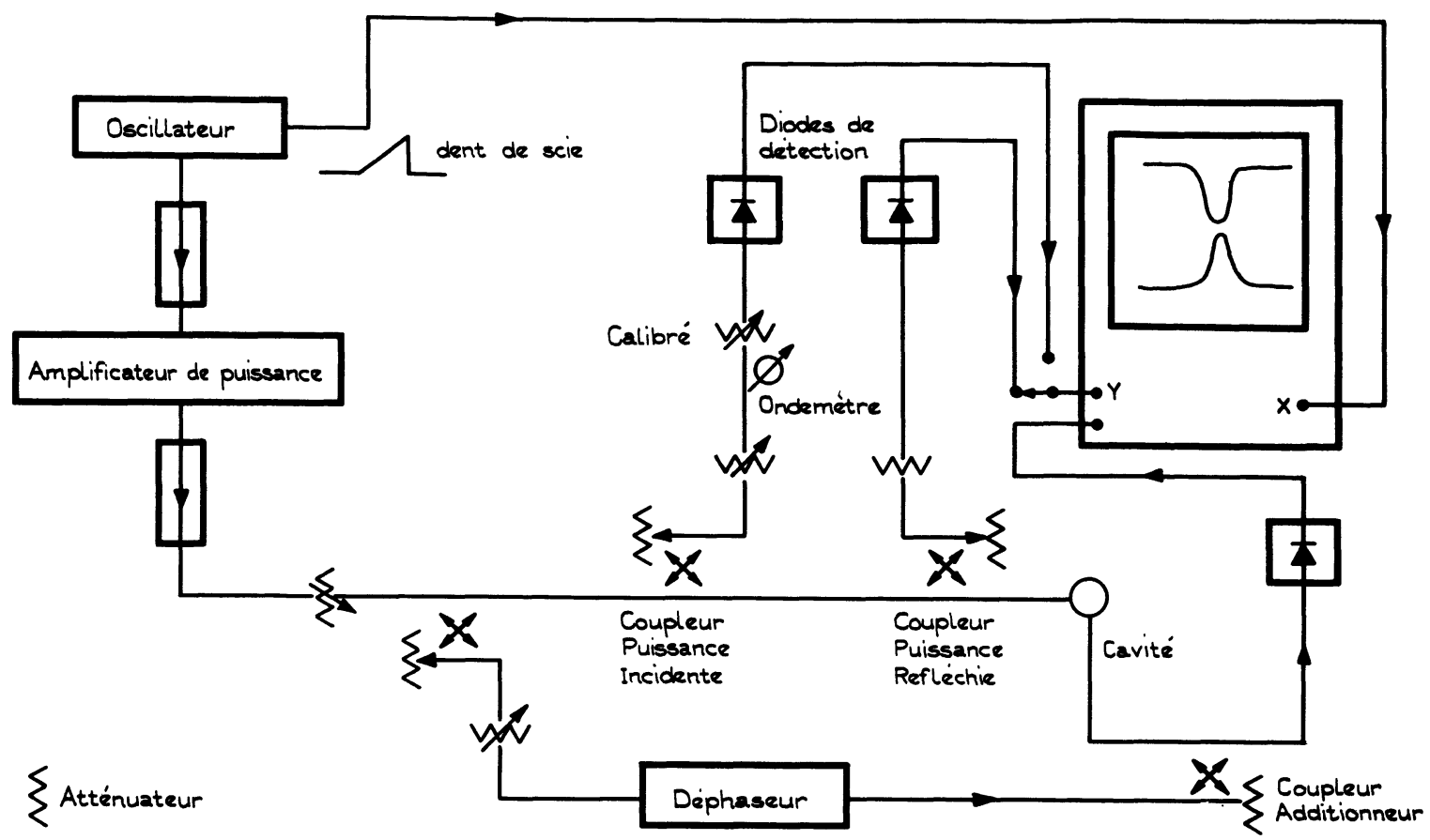

Fig. 6. - Schéma d'ensemble du montage de mesure de la mobilité.

[Block diagram for mobility measurements.]

Le second membre de cette équation peut être mesuré à l'aide de la cavité rectangulaire fonctionnant à $14 \mathrm{GHz}$ (voir $\S 2.1$ ), d'où on peut réduire $Q_{0}$ et ensuite $\Gamma_{0}$, le dernier terme inconnu donné par l'équation :

$$
\Gamma_{0}=\frac{\Gamma_{1}+Q_{1}\left(\frac{1}{Q_{1}}-\frac{1}{Q_{0}}\right)}{1-Q_{1}\left(\frac{1}{Q_{1}}-\frac{1}{Q_{0}}\right)}
$$

3.2 Détails eXPérimentauX. - La figure $5 b$ présente une vue schématique de la cavité qui est cylindrique. Les dimensions ont été choisies pour une fréquence de résonance de $14,2 \mathrm{GHz}$ dans le mode TE 111 avec un rapport bien déterminé entre le rayon et la longueur, de telle sorte que la fréquence du mode TM 011 soit suffisamment éloignée pour qu'il n'y ait pas d'interférence [21]. Le couplage « cavité-connecteur extérieur " (ici coaxial) est de type électrique et est réalisé par des embases miniatures SMA. Le couplage critique est obtenu en ajustant la longueur de l'âme centrale pénétrant dans la cavité. Les vis micrométriques permettent, en réglant la profondeur des pointes métalliques dans la cavité, de corriger les dissymétries éventuelles, en particulier celles provenant de l'échantillon. $V_{1}$ et $V_{3}$ permettent d'aligner le mode principal vers l'entrée alimentant la cavité, la puissance de sortie $\boldsymbol{P}_{\mathbf{2}}$ devant alors théoriquement s'annuler. $V_{2}$ et $V_{4}$ agissent chacune sur la fréquence de résonance de l'un des deux modes orthogonaux.

La figure 6 présente le schéma d'ensemble du montage utilisé, permettant la mesure des coefficients de réflexion à vide et avec échantillon, et le rapport des puissances transmise et incidente. L'électroaimant n'y est pas représenté. Le travail fondamental à effectuer est d'égaliser les fréquences des deux modes et de les rendre orthogonaux. Ces deux modes peuvent exister simultanément, même en l'absence de champ magnétique comme le montre la figure 7. Le pic d'importante absorption, visible dans la courbe montrant la variation de la puissance réfléchie en fonction de la fréquence, correspond bien à l'un des deux pics observés dans la variation de la puissance transmise : la fréquence centrale est la même. C'est le mode fondamental qui est fortement couplé à l'entrée. Le second pic, observable seulement dans la transmission, correspond à un mode orthogonal au premier et très faiblement couplé à l'entrée. Son existence provient des légères dissymétries de la cavité et de l'échantillon. Le rapport entre ces modes est toujours supérieur à $30 \mathrm{~dB}$ et c'est pour cela que, seul, le « fondamental » est observable dans la courbe de réflexion. Généralement, il n'est pas possible d'annuler complètement le signal transmis après orthogonalisation et dégénérescence (Fig. 7b).

Avant l'application du champ magnétique continu, on fait le "zéro " pour la fréquence de résonance en additionnant, à l'aide d'un coupleur, le signal transmis $P_{2}$ avec une partie du signal d'entrée dont on peut faire varier l'amplitude et la phase (Fig. 6).

Notons que les équations (27) ou (28) donnant la mobilité ne sont valables que pour des champs magnétiques faibles; aussi, il est nécessaire de contrôler la linéarité de $\left(P_{2} / P_{1}\right)^{1 / 2}$ en fonction de $B$. 
(a)

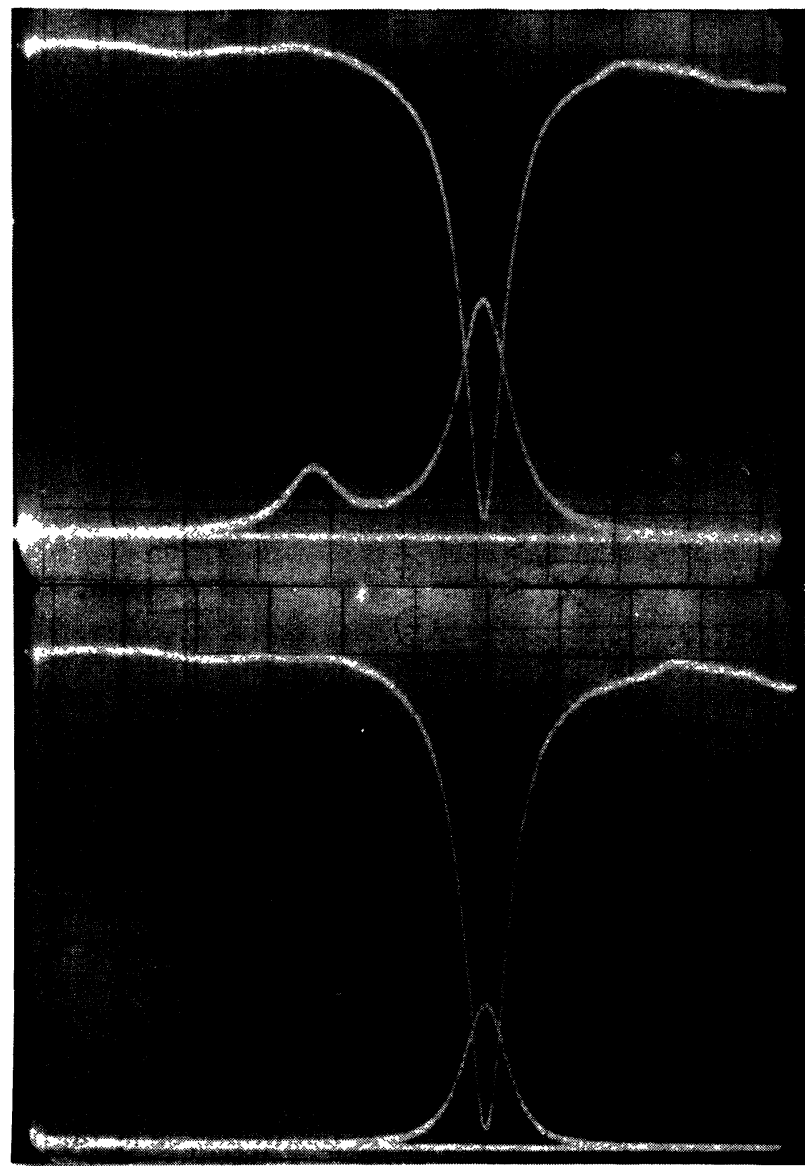

Fig. 7. - Réglage de la cavité : puissance réfléchie (courbe du haut) et puissance transmise (courbe du bas) : $a$ ) avant réglage; $b$ ) après orthogonalisation et dégénérescence. Abscisse : $15 \mathrm{MHz}$ par division ; ordonnée : échelle arbitraire avec un rapport de $40 \mathrm{~dB}$ environ entre les puissances réfléchie et transmise.

[Cavity tuning : reflected power (top) and transmitted power (bottom); a) before tuning; b) after tuning. Abscissa : $15 \mathrm{MHz} /$ div.; ordinate : arbitrary scale with about a ratio of $40 \mathrm{~dB}$ between the transmitted and reflected powers.]

4. Domaine de mesures et résultats expérimentaux. - Du fait que les mesures à vide et avec échantillon peuvent être effectuées sans démontage des cavités, la limite du domaine d'étude des faibles conductivités est fixée uniquement par les performances du matériel utilisé. Ainsi par exemple, avec la cavité coaxiale de coefficient de qualité à vide voisin de 900 , et en utilisant le synthétiseur de fréquence Hewlett Packard 8660 dont la résolution est de quelques hertz, il est possible de mesurer des variations du facteur de qualité $\left(\frac{1}{Q_{1}}-\frac{1}{Q_{0}}\right)$ de l'ordre de $10^{-6}$. La précision de mesure, dans ce cas, est limitée par les performances des microvoltmètres continus actuels. Nous avons pu déduire ainsi une valeur limite de la conductivité d'un substrat massif semi-isolant d'arséniure de gallium RT 512 :

$$
\sigma<1,3 \times 10^{-6} \Omega^{-1} \mathrm{~cm}^{-1} \text { à } 560 \mathrm{MHz} .
$$

Vers les hautes conductivités, l'utilisation de cette méthode de mesure est principalement limitée par l'amplitude de la perturbation apportée par l'échantillon. Il est raisonnable de prendre :

$$
\left(\frac{1}{Q_{1}}-\frac{1}{Q_{0}}\right) \text { et } \frac{F_{1}-F_{0}}{F_{0}}<10^{-2} .
$$

La condition concernant l'épaisseur de la couche mince (de l'ordre du micron), qui doit être bien inférieure à la profondeur d'effet de peau dans l'échantillon, est toujours réalisée.

Les variations de la fréquence de résonance et du facteur de qualité, exprimées par l'équation (7), sont illustrées en figure 8 . On constate que $\left(\frac{1}{Q_{1}}-\frac{1}{Q_{0}}\right)$ passe par un maximum, puis décroît ensuite vers zéro pour $\varepsilon_{2}^{\prime \prime}$ croissant, la perturbation ne se manifestant uniquement que par la dérive en fréquence dépendant de $\varepsilon_{2}^{\prime \prime}$ mais aussi de $v_{2}$ et $L_{2}$. La limite supérieure du domaine de mesures dépend donc de ces trois paramètres.

Le domaine de mesure de la mobilité est assez semblable au précédent, En effet, $\mu$ qui peut être exprimée par l'équation suivante dans le cas d'une symétrie parfaite :

$$
\mu=\frac{1}{B} \frac{\left[\left(\frac{1}{Q_{1}}-\frac{1}{Q_{0}}\right)+\frac{1}{Q w}+\frac{1}{Q x}\right]^{2} Q x}{2\left(\frac{1}{Q_{1}}-\frac{1}{Q_{0}}\right)}\left(\frac{P_{2}}{P_{1}}\right)^{1 / 2},
$$

où $Q x$ et $Q w$ sont des caractéristiques connues de la cavité, peut être mesurée si l'on connaît $\left(\frac{1}{Q_{1}}-\frac{1}{Q_{0}}\right)$ et

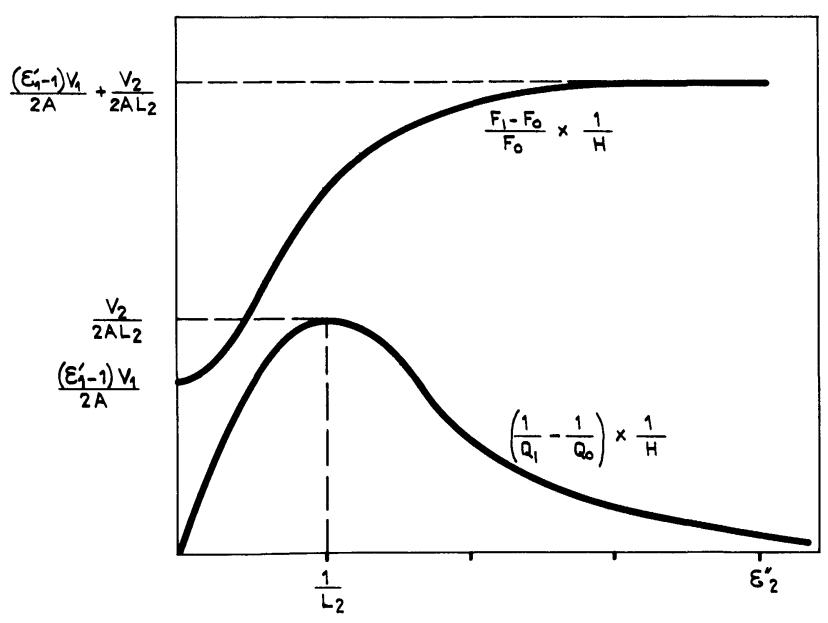

Fig. 8. - Variations de la perturbation en fonction de $\varepsilon_{2}^{\prime \prime}$. Les échelles utilisées sont linéaires. On a pris arbitrairement : $\frac{\left(\varepsilon_{1}^{\prime}-1\right) v_{1}}{2 A}<\frac{v_{2}}{2 A L_{2}}$.

[Perturbation variations as a function of $\varepsilon_{2}^{\prime \prime}$. Used scales are linear. We have arbitrarily chosen : $\left.\frac{\left(\varepsilon_{1}^{\prime}-1\right) v_{1}}{2 A}<\frac{v_{2}}{2 A L_{2}} \cdot\right]$ 
$P_{2}$. Le domaine de mesure de la variation du facteur de qualité vient d'être défini et celui de $P_{2}$ ne dépend que des performances du système de détection [22]. Pour ce travail, nous avons utilisé un système de détection classique de signal modulé en amplitude permettant des mesures du rapport $P_{2} / P_{1}$ aussi faibles que $10^{-6}$.

Les résultats présentés au tableau I concernent deux couches minces obtenues par jet moléculaire, sur lesquelles il a été effectué des mesures d'effet Hall et de conductivité à température ambiante, d'abord en continu, puis en hyperfréquence. Nous avons reporté uniquement les valeurs de $\sigma$ satisfaisant à la condition $L_{2} \varepsilon_{2}^{\prime \prime} \gg 1$ ou $\ll 1$, c'est-à-dire celles obtenues, soit à $560 \mathrm{MHz}$, soit à $14 \mathrm{GHz}$ selon l'échantillon à mesurer. En effet, comme cela a déjà été vu au $\S 2.1$, le terme : $A=1+L_{1}\left(\varepsilon_{1}^{\prime}-1\right)$ de l'équation (9) n'est pas connu exactement car la valeur du facteur de dépolarisation considéré est celle d'un ellipsoïde et non d'une plaquette. On estime que la valeur du facteur de dépolarisation déduite expérimentalement est environ $15 \%$ supérieure à celle déduite des courbes d'Osborn [10]. Cependant, l'erreur sur $\sigma$ ne doit pas dépasser $50 \%$ dans la zone la plus défavorable, c'est-à-dire quand $L_{2} \varepsilon_{2}^{\prime \prime}$ est voisin de 1 . Si les valeurs de la conductivité obtenues aux deux fréquences de mesure diffèrent d'un facteur supérieur à 1,5 , il faut incriminer la présence d'un mécanisme de conduction ne satisfaisant pas à la condition $\omega \tau \ll 1[4]$, où $\tau$ est le temps de relaxation.

Dans le cas présent, il faut noter l'accord très satisfaisant entre les résultats obtenus en continu et en hyperfréquence, malgré l'incertitude existant sur le facteur de dépolarisation. Cet accord serait encore meilleur si on prenait la valeur expérimentale de $L_{1}$ qui peut être mesurée sur un substrat semi-isolant de même nature et de même forme. Le fait que cette méthode de mesure est encore utilisable quand le champ dépolarisant dû aux porteurs libres est très grand $\left(L_{2} \varepsilon_{2}^{\prime \prime} \sim 20\right)$ est très encourageant pour envisager l'étude de couches très minces et très conductrices.

En ce qui concerne les mesures de mobilité, l'accord est très satisfaisant, vu que la précision est estimée à $\pm 20 \%$. La figure 9 illustre la linéarité du rapport

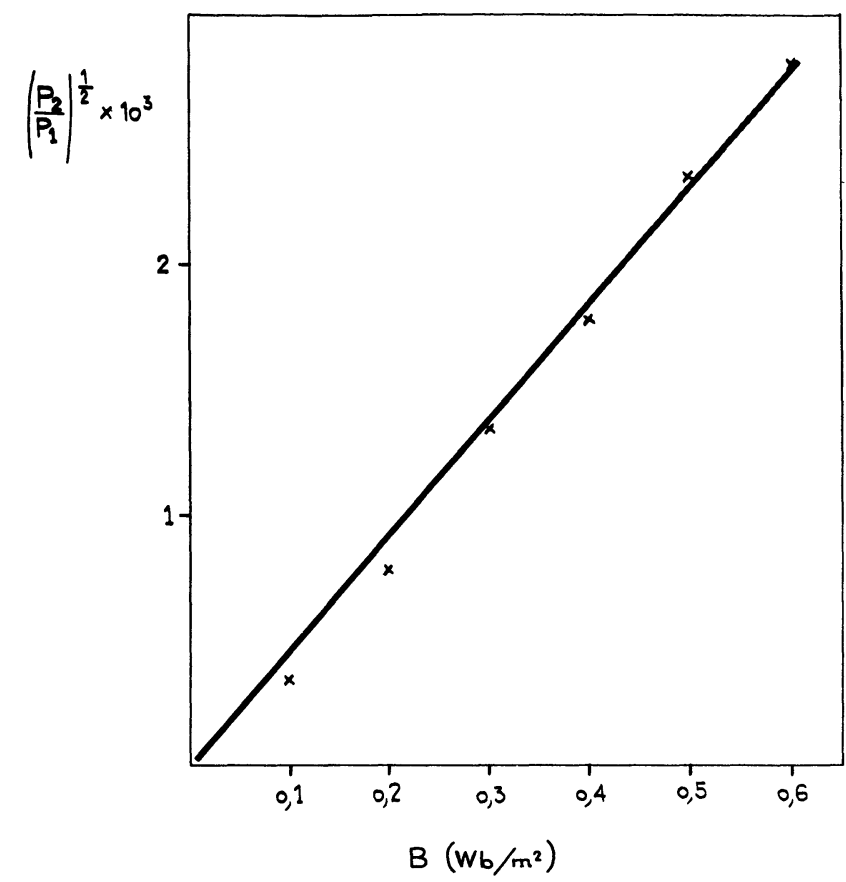

Fig. 9. - Linéarité de $\left(P_{2} / P_{1}\right)^{1 / 2}$ en fonction du champ magnétique continu pour l'échantillon le plus conducteur (MBE a).

[Linearity of $\left(P_{2} / P_{1}\right)^{1 / 2}$ versus the static magnetic field for the most conducting sample.]

$\left(P_{2} / P_{1}\right)^{1 / 2}$ en fonction de $B$ pour la couche la plus conductrice. Notons que la valeur de $P_{2}$ n'est pas modifiée si $B$ est inversé.

5. Conclusion - La chaîne de mesure décrite ici est bien adaptée pour caractériser sans destruction les couches minces conductrices créées à partir d'un substrat isolant. Du fait que les échantillons, qui se présentent sous forme de plaquettes, peuvent être introduits dans la cavité sans démontage mécanique, il est possible de mesurer des faibles variations du facteur de qualité, et donc d'étendre le domaine de mesure vers les basses conductivités, d'une part, et, ce qui nous intéresse le plus, vers les fortes valeurs.

Tableau I. - Valeurs de la mobilité et de la conductivité en continu et en hyperfréquence pour deux couches minces déposées sur substrat semi-isolant. Ici, n est le nombre de porteurs par $\mathrm{cm}^{3}$ et e la charge de l'électron.

[D.C. and microwave values of the mobility and the conductivity for two thin films made on semi-insulating substrate; $n$ represents the carrier concentration and $e$ the electron charge.]

\begin{tabular}{|c|c|c|c|c|c|c|c|c|c|}
\hline \multirow{2}{*}{ Echantillon } & \multirow{2}{*}{$\begin{array}{c}\mu_{\text {continu }} \\
\mathrm{cm}^{2} / \text { V.s }\end{array}$} & \multirow{2}{*}{$\begin{array}{r}\mu_{14 \mathrm{GHz}} \\
\mathrm{cm}^{2} / \mathrm{V} . \mathrm{s}\end{array}$} & \multicolumn{3}{|c|}{$n_{\mathrm{cm}-3}=\frac{\sigma}{e \mu}$} & \multicolumn{2}{|c|}{$L_{2} \varepsilon_{2}^{\prime \prime}$} & \multirow{2}{*}{$\begin{array}{c}\sigma \\
\Omega^{-1} \cdot \mathrm{cm}^{-1}\end{array}$} & \multirow{2}{*}{$\begin{array}{l}\text { Epaisseur } \\
\text { de la couche }\end{array}$} \\
\hline & & & continu & $560 \mathrm{MHz}$ & $14 \mathrm{GHz}$ & $560 \mathrm{MHz}$ & $14 \mathrm{GHz}$ & & \\
\hline MBE a & 570 & 560 & $9,5 \times 10^{17}$ & $8,5 \times 10^{17}$ & & 18,5 & 0,75 & 77 & $3,6 \mu$ \\
\hline MBE b & 2800 & 3300 & $1,39 \times 10^{16}$ & & $1,1 \times 10^{16}$ & 0,63 & 0,025 & 5,8 & $0,25 \mu$ \\
\hline
\end{tabular}


La limite supérieure, définie $\operatorname{par}\left(F_{1}-F_{0}\right) / F_{0}<10^{-2}$, dépend de $\varepsilon_{2}^{\prime \prime}$ et du facteur de dépolarisation $L_{2}$ de la couche mince. Par contre, la mesure de la mobilité est d'autant plus aisée que sa valeur est élevée pour une perturbation donnée. Mise à part l'erreur systématique sur le facteur de dépolarisation, on estime que la précision de mesure de $\sigma$ et de $\mu$ est meilleure que $20 \%$ compte tenu, d'une part, de l'erreur provenant de l'appréciation des dimensions, en particulier de l'épaisseur de l'échantillon, et, d'autre part, de l'imprécision des mesures. La possibilité de pouvoir mesurer $\sigma$ à deux fréquences très différentes est très importante : elle permet d'améliorer la précision de mesure quand l'incertitude sur le facteur de dépolarisation prend une grande importance et surtout de déceler la présence éventuelle d'un mécanisme de conduction indésirable $(\omega \tau \sim 1)$.

Remerciements. - Nous tenons à remercier Messieurs Castelletto, Crespel, Goloubkoff, du C.N.E.T., et Truffin, de l'Université de Rennes, pour leur contribution à la réalisation des différents montages mécaniques et de l'amplificateur hyperfréquence. Nous remercions également Messieurs Dupas, Palmier et Regreny pour la fourniture des échantillons obtenus par jet moléculaire.

\section{Bibliographie}

[1] Handbook of Microwave Measurements, Polytechnic Press of the Polytechnic Institute of Brooklyn (John Wiley and Sons) 1963, vol. II.

[2] Works, C. N., Dakin, T. W. et Boggs, F. W., Proc. I.R.E. (avril 1945) 245.

[3] Portis, A. M., Dale, T., J. Appl. Phys. 29 (1958) 1692.

[4] Le Cleac'h, X., J. Physique 40 (1979) 417.

[5] Sayed, M. M., Wesgate, C. R., Perlstein, J. H., Electron. Lett. 9 (1973) 529.

[6] Crowley, J. D., Rabson, T. A., Rev. Sci. Instrum. 47 (1976) 712.

[7] Miller, G. L., Robinson, D. A. H., Wiley, J. D., Rev. Sci. Instrum. 47 (1976) 799.

[8] Référence [1], page 533.

[9] ShChegolev, I. F., Phys. Status Solidi (a) 12 (1972) 9.

[10] Osborn, J. A., Phys. Rev. 67 (1945) 351.

[11] Favennec, P. N., Henry, L., L'Haridon, H., Solid State Electron. 21 (1978) 705.

[12] Esaki, L., Chang, L. L., Thin Solid Films 36 (1976) 285.
[13] Birnbaum, G. et Franeau, J., J. Appl. Phys. 20 (1949) 817.

[14] Slater, J. C., Microwave Electronics (D. Van Nostrand, Inc. Canada) 1950, page 233.

[15] Liu, S. H., Nishina, Y. et Good, R. H., Rev. Sci. Instrum. 32 (1961) 784.

[16] Watanabe, N., Rev. Electrical Commun. Lab. 8 (1960) 256.

[17] Slater, J. C., Rev. Mod. Phys. 18 (1946) 441.

[18] Watanabe, N., J. Phys. Soc. Japan 16 (1961) 1979.

[19] SAYED, M. et Westgate, R., Rev. Sci. Instrum. 46 (1975) 1074.

[20] Référence [1], page 451.

[21] PoOle, C. P., Electron Spin Resonance (Interscience Publishers, John Wiley and Sons) 1967, pages 270, 271.

[22] Sayed, M. M. et Westgate, R., Rev. Sci. Instrum. 46 (1975) 1080. 